\title{
Early Daytime Sleepiness and Nighttime Slow-wave Sleep Disorganization in Progressive Macaque Model of Parkinson's Disease
}

\author{
Aurélie Davin \\ Clinatec \\ Stéphan Chabardès \\ Grenoble Alpes University \\ Hayat Belaid \\ Pitié-Salpêtrière Hospital \\ Daniel Fagret \\ Grenoble Alpes University \\ Loic Djaileb \\ Grenoble Alpes University \\ Yves Dauvilliers \\ University of Montpellier \\ Olivier David \\ Aix-Marseille University \\ Napoléon Torres-Martinez \\ Clinatec
}

Brigitte Piallat ( $\square$ brigitte.piallat@univ-grenoble-alpes.fr)

Grenoble Alpes University

\section{Research Article}

Keywords: Parkinson's disease, Early Wake/Sleep disorders, Biomarker, Non-human primate

Posted Date: January 11th, 2022

DOI: https://doi.org/10.21203/rs.3.rs-1216338/v1

License: @ (1) This work is licensed under a Creative Commons Attribution 4.0 International License. Read Full License 


\section{Abstract}

Parkinsonian patients often experience wake/sleep behavior disturbances, which can appear at an early stage of the disease in a way that is still not fully described. We aimed here at reproducing and characterizing these clinical signs in a progressive non-human primate model of the Parkinson's disease to better understand the underlying physiopathology and to identify biomarkers of the disease. Three adult non-human primates (macaca fascicularis) were equipped with a polysomnographic telemetry system allowing the characterization of the wake/sleep behavior by long-term neurophysiological recordings and a modified multiple sleep latency test. Experiments were first performed in healthy animals and then during the progressive induction of a parkinsonian syndrome by chronic intramuscular injections of low doses of MPTP. We observed a significant early onset of wake/sleep behavior disturbances, before any motor symptoms, resulting in (i) a disorganization of nighttime sleep with more deep sleep and (ii) a disorganization of daytime naps with an excessive daytime sleepiness characterized by longer duration of naps, which occurred faster. These observations persisted and worsened in stable symptomatic state. In that latter state, we observed persistent excessive daytime sleepiness and more disorganized nighttime sleep architecture and continuity. Interpolating to the human condition, the present study suggests that nighttime and daytime sleep disorders may appear in early stage of the disease. They could thus be used as biomarkers of the disease for early stratification of patients who are at risk of developing Parkinson's disease.

\section{Significance Statement}

Wake and sleep disturbances are very common symptoms of Parkinson's disease that strongly affect patients' independence. These disorders seem to appear before motor symptoms, but their precise timing of onset as well as their nature are not yet well established. Here, we studied the kinetics of appearance of wake and sleep disorders in a progressive macaque model of Parkinson's disease. This approach allowed us to show that excessive daytime sleepiness, associated with nighttime sleep disorganization, appears at an early stage of the disease, while motor symptoms are not yet detectable. This study suggests that these wake/sleep disorders, which are easily detectable, deserve to be monitored and could therefore be used as biomarkers of the disease.

\section{Introduction}

With a prevalence of $60-70 \%$, wake/sleep (W/S) disorders are one of the most common non-motor symptoms (NMS) in Parkinson's disease (PD) and lead to decrease significantly the quality of life of patients ${ }^{1,2}$. Rapid-eye movement (REM) sleep behavior disorders, insomnia and an increase in nighttime awakenings $^{3-5}$ are frequently met in patients. Also, altered wakefulness is often reported, characterized by excessive daytime sleepiness (EDS) ${ }^{1,6,7}$. Furthermore, some patients exhibit EDS with sleep attacks, which can be constituted by REM sleep, as in narcolepsy ${ }^{6,8,9}$. These signs, initially attributed to the motor impairments or dopaminergic medications, illustrate a total disorganization of circadian rhythm ${ }^{10}$ and may be an intrinsic feature of the disease itself. Indeed, the degenerative process in PD mainly affects the dopaminergic neurons of the substantia nigra which may induce dysfunction of other brain structures known to be involved in W/S behavior such as the noradrenergic locus cœruleus ${ }^{11}$, the cholinergic pedunculopontine nucleus ${ }^{12}$ and the orexin hypothalamic $^{13,1413,14}$ systems. Moreover, these structures also seem to be affected by neurodegenerative processes which may explain the worsening of W/S disorders over time. Interestingly, several studies have shown that W/S disorders, mostly REM sleep behavior disorders and EDS, may precede motor symptoms by many years suggesting that they could be used as biomarkers of PD 1,8,15. At this time, RBDs are the most widely used NMSs as biomarker of $\mathrm{PD}^{15}$. Also, multiple changes are observed during REM sleep ranging from behavioural observations to biological constants such as heart rate variability. Indeed, heart rate variability is known to be high in normal REM sleep compare to other sleep stages ${ }^{16}$ and seems to decrease in PD patients with REM sleep behavior disorders ${ }^{17}$. Although the link between sleep and wakefulness disorders is still controversial, studies have shown that nighttime sleep disorders and EDS are unrelated ${ }^{6,18}$. But the frequency and time of appearance of these specific NMSs relative to the first motor symptoms have not been fully determined. As growing evidences showed that the sleep seems more and more involved in the metabolite clearance from the brain ${ }^{19,20}$, the study of the kinetic of appearance of the W/S disturbances, could be crucial given the restorative and regenerative effects of sleep ${ }^{21,22}$, suggesting that their earlier management could have an impact on improving the quality of life of PD patients and even on slowing the disease's progression.

1-Methyl-4-phenyl-1,2,3,6-tetrahydropyridine (MPTP)-treated non-human primate (NHP) is recognized as a model of choice to study PD due to the similarities with parkinsonian patients in term of brain damages and symptoms ${ }^{23,24}$. Some studies have reported circadian rhythm disorders, but were only based on actimetry techniques ${ }^{25,26}$. Very few studies, using polysomnographic techniques that provide objective analysis of the W/S stages, described severe nighttime sleep alterations ${ }^{27-29}$. They also reported daytime sleepiness but their occurrence and nature have never been precisely described. Furthermore, all the above mentioned studies did not have investigated the prodromal phase i.e. before motor symptoms appearance. In our study, we report, for the first time, the nature of nighttime alterations and, especially, of daytime sleepiness in a prodromal phase, using a progressive model of PD requiring 4 to 6 months of intoxication. These results have significant implications for (i) the use of chronically MPTP-treated NHPs to develop news therapeutic strategies to treat W/S disorders and (ii) the use of specific W/S disorders as biomarkers of PD for both diagnosed earlier and open a window for neuroprotective strategy.

\section{Results}

\section{Chronic low doses of MPTP induces slow onset of motor symptoms}

Throughout the MPTP intoxication protocol, animals exhibited a gradual onset of motor symptoms which allows to identify several keys periods. First of all, animals went through a presymptomatic period, lasting from 4 to 6 months characterized by no visible motor symptoms (starting just after the first MPTP injection) (Fig. 1.B). Next, a period of stabilization of the parkinsonian state characterized by the appearance of slight and unstable motor symptoms started (Fig. 1.A). Data collected during this fluctuating period were excluded from analyzes. Finally, animals exhibited a stable parkinsonian syndrome, after a cumulative dose of $5.2 \pm 1.7 \mathrm{mg} / \mathrm{kg}$ (motor score of $16.4 \pm 1.5$ ), for several months without observation of recovery phenomena. This parkinsonian state was characterized by a decrease in general activity (Fig. 1.C), flexed posture, bradykinesia evolving into akinesia and action tremors. The condition of M2 was more 
severe, with fewer injections of MPTP than M1 and M3, but he was still able to take care of himself (Fig. 1.B). Compared to the healthy state, the spectral analysis of EMG highlighted a significantly higher power density in the symptomatic state and a tendency to be higher in the presymptomatic state (Fig. 1.E). In healthy and presymptomatic animals, spectral analysis of EEG recordings during active wake was composed of low power density of mixed-frequency. When the animals were symptomatic, a significant peak appeared in the low beta band at $13 \mathrm{~Hz}$ (Fig. 1.D).

Nighttime sleep disorganization: evolution from presymptomatic to symptomatic state

Healthy animals exhibited a similar sleep architecture with a high proportion of S3 in the first part of the night and a greater proportion of REM sleep in the second part of the night (Fig. 2.A). Healthy animals had an average of eleven well-defined sleep cycles which had begun to decrease from the presymptomatic state in M2 and M3 and significantly decrease in the three animals in the symptomatic state (Table 1.A). In the presymptomatic state, M1 and M2 spent more time in S3 rather than in S1 and S2 (25.2 $\pm 1.5 \%$ : healthy vs $39.6 \pm 1.1 \%$; $p=0.0212$ : presymptomatic for M1 and $18.2 \pm 1.7 \%$ : healthy vs $34.0 \pm 2.3 \%$; $p=0.0184$ : presymptomatic for M2) (Table 1.A). Changes in EEG power density were observed in the delta frequency band (0.3-4Hz) during S3, i.e. deep sleep. Indeed, from the presymptomatic state, there was a significant decrease in power density for the delta band despite an increase in its quantity compared to the healthy state (Fig. 2.D). For M1 and M2 animals, the TST and WASO did not show any significant changes which does not lead to any changes in sleep efficiency. However, M2 showed a significant decrease in REM sleep during this period ( $11.4 \pm 0.7 \%$ : healthy vs $4.5 \pm 0.8 \%$; $p=0.0007$ : presymptomatic). M3 did not present an increase in S3 in the presymptomatic state but presented a progressive alteration in all sleep parameters (Table 1.A). 
Table 1

Sleep parameters from long-term $12 \mathrm{~h}$ recordings of nighttime and daytime for each animal in healthy, presymptomatic and symptomatic

\begin{tabular}{|c|c|c|c|c|c|c|c|c|}
\hline \multirow[t]{3}{*}{ (A) } & \multicolumn{8}{|c|}{ NIGHTTIME } \\
\hline & \multicolumn{3}{|l|}{ M1 } & \multicolumn{3}{|l|}{ M2 } & \multicolumn{2}{|l|}{ M3 } \\
\hline & Healthy & Presymptomatic & Symptomatic & Healthy & Presymptomatic & Symptomatic & Healthy & Presymptomatic \\
\hline $\mathrm{SL}(\min )$ & $\begin{array}{l}13.4 \pm \\
1.5\end{array}$ & $22.2 \pm 1.5$ * & $18.7 \pm 2.8$ & $\begin{array}{l}7.5 \pm \\
1.2\end{array}$ & $0.3 \pm 0.3$ * & $4.8 \pm 1.5$ & $\begin{array}{l}10.2 \pm \\
1.6\end{array}$ & $17.3 \pm 2.1$ \\
\hline$\%$ AW & $\begin{array}{l}17.2 \pm \\
0.8\end{array}$ & $19.0 \pm 0.6$ & $38.0 \pm 1.3$ * \# & $\begin{array}{l}15.5 \pm \\
1\end{array}$ & $12.8 \pm 0.9$ & $28.0 \pm 2.1^{*} \#$ & $\begin{array}{l}20.2 \pm \\
0.8\end{array}$ & $21.7 \pm 1.1$ \\
\hline$\% \mathrm{~W}$ & $\begin{array}{l}10.0 \pm \\
0.8\end{array}$ & $11.8 \pm 0.6$ & $29.0 \pm 1.2$ * \# & $\begin{array}{l}2.7 \pm \\
0.5\end{array}$ & $2.9 \pm 0.8$ & $13.6 \pm 2.3$ * \# & $\begin{array}{l}8.8 \pm \\
0.5\end{array}$ & $13.8 \pm 0.9$ * \\
\hline $\begin{array}{l}\% \text { Stage } \\
1\end{array}$ & $\begin{array}{l}18.2 \pm \\
1.0\end{array}$ & $10.2 \pm 0.5$ * & $13.3 \pm 1.1$ *\# & $\begin{array}{l}20.0 \pm \\
2.0\end{array}$ & $13.8 \pm 2.0$ & $31.5 \pm 0.9$ * \# & $\begin{array}{l}16.9 \pm \\
1.6\end{array}$ & $22.1 \pm 1.5$ \\
\hline $\begin{array}{l}\% \text { Stage } \\
2\end{array}$ & $\begin{array}{l}17.0 \pm \\
2.0\end{array}$ & $5.2 \pm 0.5$ * & $8.2 \pm 0.4$ *\# & $\begin{array}{l}32.1 \pm \\
2.2\end{array}$ & $32.2 \pm 1.6$ & $14.6 \pm 2.0$ * \# & $\begin{array}{l}24.0 \pm \\
1.3\end{array}$ & $17.7 \pm 1.3$ * \\
\hline $\begin{array}{l}\% \text { Stage } \\
3\end{array}$ & $\begin{array}{l}25.2 \pm \\
1.5\end{array}$ & $39.6 \pm 1.1$ * & $5.4 \pm 0.6$ * \# & $\begin{array}{l}18.2 \pm \\
1.7\end{array}$ & $34.0 \pm 2.3$ * & $5.1 \pm 0.9$ * \# & $\begin{array}{l}18.6 \pm \\
1.2\end{array}$ & $15.2 \pm 1.3$ \\
\hline $\begin{array}{l}\% \text { REM } \\
\text { sleep }\end{array}$ & $\begin{array}{l}12.4 \pm \\
0.4\end{array}$ & $14.3 \pm 0.6$ & $5.1 \pm 0.4$ * \# & $\begin{array}{l}11.4 \pm \\
0.7\end{array}$ & $4.5 \pm 0.8$ * & $7.1 \pm 0.7$ * \# & $\begin{array}{l}10.7 \pm \\
0.4\end{array}$ & $9.5 \pm 0.5$ \\
\hline $\begin{array}{l}\text { TST } \\
\text { (min) }\end{array}$ & $\begin{array}{l}523.1 \pm \\
5.1\end{array}$ & $498.8 \pm 6.3$ & $\begin{array}{l}230.4 \pm 7.0 \text { * } \\
\#\end{array}$ & $\begin{array}{l}589.4 \pm \\
9.3\end{array}$ & $607.3 \pm 11.4$ & $\begin{array}{l}419.9 \pm 14.0 \\
* \#\end{array}$ & $\begin{array}{l}505.2 \pm \\
7.9\end{array}$ & $468.4 \pm 10.3$ * \\
\hline $\begin{array}{l}\text { WASO } \\
\text { (min) }\end{array}$ & $\begin{array}{l}182.3 \pm \\
5.2\end{array}$ & $197.4 \pm 6.2$ & $\begin{array}{l}465.8 \pm 6.7 \text { * } \\
\#\end{array}$ & $\begin{array}{l}120.1 \pm \\
9.3\end{array}$ & $106.6 \pm 11.6$ & $\underset{* \#}{289.4 \pm 13.3}$ & $\begin{array}{l}199.8 \pm \\
8.0\end{array}$ & $234.9 \pm 6.3$ * \\
\hline $\begin{array}{l}\text { Sleep } \\
\text { efficiency } \\
(\%)\end{array}$ & $\begin{array}{l}72.7 \pm \\
0.7\end{array}$ & $70.2 \pm 0.9$ & $32.0 \pm 1.0$ * \# & $\begin{array}{l}81.9 \pm \\
1.3\end{array}$ & $84.3 \pm 1.6$ & $58.3 \pm 1.9$ * \# & $\begin{array}{l}70.2 \pm \\
1.1\end{array}$ & $64.5 \pm 0.8$ * \\
\hline $\begin{array}{l}\text { Sleep } \\
\text { cycle } \\
\text { number }\end{array}$ & $\begin{array}{l}11.4 \pm \\
0.3\end{array}$ & $10.3 \pm 0.4$ & $0.8 \pm 0.4$ * \# & $\begin{array}{l}11.4 \pm \\
0.6\end{array}$ & $6.8 \pm 0.9$ * & $5.7 \pm 0.7$ * & $\begin{array}{l}10.6 \pm \\
0.3\end{array}$ & $8.7 \pm 0.3$ * \\
\hline \multirow[t]{3}{*}{ (B) } & \multicolumn{8}{|l|}{ DAYTIME } \\
\hline & M1 & & & M2 & & & M3 & \\
\hline & Healthy & Presymptomatic & Symptomatic & Healthy & Presymptomatic & Symptomatic & Healthy & Presymptomatic \\
\hline $\mathrm{SL}(\mathrm{min})$ & $\begin{array}{l}41.1 \pm \\
6.1\end{array}$ & $25.8 \pm 4.8$ & $8.7 \pm 2.6$ * & $\begin{array}{l}55.0 \pm \\
7.2\end{array}$ & $28.6 \pm 5.2$ & $16.6 \pm 6.4$ * & $\begin{array}{l}77.3 \pm \\
17.8\end{array}$ & $67.8 \pm 16.3$ \\
\hline $\begin{array}{l}\% \text { Stage } \\
1\end{array}$ & $\begin{array}{l}8.6 \pm \\
1.1\end{array}$ & $6.6 \pm 0.5$ & $11.0 \pm 0.8 \#$ & $\begin{array}{l}6.0 \pm \\
0.9\end{array}$ & $12.3 \pm 2.0$ & $18.4 \pm 1.2$ * & $\begin{array}{l}3.0 \pm \\
0.4\end{array}$ & $4.3 \pm 0.7$ \\
\hline $\begin{array}{l}\% \text { Stage } \\
2\end{array}$ & $\begin{array}{l}5.0 \pm \\
0.4\end{array}$ & $4.2 \pm 0.7$ & $3.7 \pm 0.5$ & $\begin{array}{l}2.9 \pm \\
0.6\end{array}$ & $10.9 \pm 2.3$ * & $9.1 \pm 2.0$ * & $\begin{array}{l}2.3 \pm \\
0.4\end{array}$ & $2.5 \pm 0.5$ \\
\hline $\begin{array}{l}\% \text { Stage } \\
3\end{array}$ & $\begin{array}{l}1.1 \pm \\
0.3\end{array}$ & $3.2 \pm 0.5$ * & $0.2 \pm 0.1 \#$ & $\begin{array}{l}0.0 \pm \\
0.0\end{array}$ & $1.3 \pm 0.7$ * & $0.1 \pm 0.05$ & $\begin{array}{l}0.2 \pm \\
0.2\end{array}$ & $0.4 \pm 0.2$ \\
\hline $\begin{array}{l}\% \text { REM } \\
\text { sleep }\end{array}$ & $\begin{array}{l}0.2 \pm \\
0.1\end{array}$ & $1.5 \pm 0.4$ * & $1.2 \pm 0.3$ * & $\begin{array}{l}0.1 \pm \\
0.1\end{array}$ & $0.1 \pm 0.1$ & $1.0 \pm 0.2 * \#$ & $\begin{array}{l}0.1 \pm \\
0.1\end{array}$ & $0.1 \pm 0.1$ \\
\hline $\begin{array}{l}\text { TST } \\
\text { (min) }\end{array}$ & $\begin{array}{l}107.4 \pm \\
8.2\end{array}$ & $112.1 \pm 10.2$ & $115.0 \pm 7.7$ & $\begin{array}{l}64.8 \pm \\
8.1\end{array}$ & $176.3 \pm 24.3$ * & $205.6 \pm 17.7$ * & $\begin{array}{l}39.7 \pm \\
6.1\end{array}$ & $52.6 \pm 8.0$ \\
\hline$\%$ AW & $\begin{array}{l}80.6 \pm \\
1.4\end{array}$ & $76.8 \pm 2.2$ & $50.5 \pm 2.4$ * \# & $\begin{array}{l}85.8 \pm \\
1.8\end{array}$ & $69.9 \pm 3.4$ * & $61.6 \pm 2.0$ * & $\begin{array}{l}89.3 \pm \\
1.5\end{array}$ & $86.8 \pm 1.9$ \\
\hline$\% \mathrm{~W}$ & $\begin{array}{l}4.4 \pm \\
0.6\end{array}$ & $7.6 \pm 1.3$ & $27.3 \pm 1.7$ * \# & $\begin{array}{l}5.3 \pm \\
0.8\end{array}$ & $5.6 \pm 0.7$ & $9.7 \pm 1.1$ * \# & $\begin{array}{l}5.2 \pm \\
0.7\end{array}$ & $5.9 \pm 0.9$ \\
\hline
\end{tabular}

Table 1: (A) Each column represents mean values ( \pm SEM) derived from 10 healthy, 10 presymptomatic and 10 symptomatic nights for M1, M2 and M3. SL correspond to the time in minute between the light turned off $(7 \mathrm{pm})$ and the first sleep episode. Values in lines $2,3,4$ and 5 are expressed as the mean percentage of the total scoring time with $\mathrm{AW}=$ active wake and $\mathrm{W}=$ quiet wake. TST refers to the total sleep time during the night i.e. between 7 pm and 7 am. Wake time after sleep onset (WASO) is expressed in minute and refers to the sleep period time minus the TST. Sleep efficiency, expressed in percentage, was defined as the ratio of TST to the $12 \mathrm{~h}$ nocturnal time. (B) Each column represents mean values ( \pm SEM) derived from 10 healthy, 10 presymptomatic and 10 symptomatic days for M1, M2 and M3. Sleep latency (SL) refer to the first sleep after wake and TST refer to the total sleep time during the day. Each wake and sleep stages are expressed in mean percentage of the total scoring time. * $p<0.05$ : Kruskal-Wallis test followed by Dunn's multiple comparisons test in the event of statistically significant differences. 
Once parkinsonian syndrome was established, all animals showed a strongly disorganized sleep architecture (Fig. 2.A). Indeed, we observed a decrease in S3 and REM sleep accompanied by an increase in S1. In this state, changes in EEG power density during deep sleep S3 were also observed with a significant decrease in $0.3-5 \mathrm{~Hz}$ delta power and a significant increase in $8-12 \mathrm{~Hz}$ alpha power (Fig. 2.D). These changes were accompanied by a significant decrease in TST associated with an increase in WASO, which led to a significant decrease in sleep efficiency (Table 1.A). In addition, spectral analysis of the EMG during REM sleep showed a progressive increase in the amplitude of muscle tone activity from healthy to symptomatic state (Fig. 2.C). In parallel, the study of the heart rate variability during REM sleep showed that the interval interbeat (meanNN) remains stable between healthy and presymptomatic states but decreased significantly in the symptomatic state $(0.801 \pm 0.116 \mathrm{~s}$ vs $0.795 \pm 0.177 \mathrm{~s}$ vs $0.688 \pm 0.108 \mathrm{~s}$, respectively). However, we observed a more important data dispersion in the presymptomatic state characterized by a higher coefficient of variation than in the healthy and symptomatic state (22,31\% in presymptomatic state vs $14,43 \%$ in healthy state vs $15,67 \%$ in symptomatic state) (Fig. 2.E).

\section{Daytime hypersomnia assessment: evolution from presymptomatic to symptomatic state}

Healthy animals exhibited three to four naps lasting 30 to $60 \mathrm{~min}$. From presymptomatic to symptomatic states, wakefulness was increasingly fragmented by sleep episodes throughout the day (Fig. 2.B) and the first nap appeared earlier, which became significantly different in the symptomatic state for M1 and M2 (Table 1.B). M3 did not show significant changes but followed the same tendency. Interestingly, we observed that the first sleep after wake is significantly impacted before the sleep efficiency which is impacted only in symptomatic state (Fig. 2.F). In all animals, the composition of naps changed throughout the disease progression with many variations (Table 1.B). In presymptomatic state, for M1 and M2, we observed an increase in S3 compared to the healthy state which was almost abolished in the symptomatic state. M1 had the REM sleep significantly increased all along the disease progression ( $0.2 \pm 0.1 \%$ : healthy vs $1.5 \pm 0.4 \% ; p=0.0120$ : presymptomatic vs $1.2 \pm 0.3 \% ; p=0.0340$ : symptomatic). Then, dramatic changes were observed in symptomatic state compared to the healthy state, with an increase in TST and therefore a decrease of wake states (Table 1.B).

\section{Early onset of daytime sleepiness with mMSLT}

In the presymptomatic state, the MMSLT showed that M1 and M2 fell asleep significantly more rapidly than they did in the healthy state (SL: $13.5 \pm 0.9$ min: healthy vs $6.3 \pm 0.4 \mathrm{~min}$ : presymptomatic for M1 and $11.6 \pm 0.8 \mathrm{~min}$ : healthy vs $7.4 \pm 1.1 \mathrm{~min}$ : presymptomatic for M2), and M3 exhibited some sleep episodes for the first time (SL: $16 \pm 1 \mathrm{~min}$ ) (Table 2). This phenomenon was observed from the first MPTP injection. In the presymptomatic and symptomatic states, the SL was shorter than it was in the healthy state for each individual light-OFF session (example SL light-OFF session 1 for M2: $12.43 \pm 1.45 \mathrm{~min}$ : healthy vs 7.17 $\pm 1.73 \mathrm{~min}$ : presymptomatic vs $4.61 \pm 1.59 \mathrm{~min}$ : symptomatic) except light-OFF session 3 for M2 (Fig. 3.A). M1 and M2 presented a significant increase in time spent sleeping in the presymptomatic and symptomatic states compared to the healthy state (Fig. 3.B). M3 did not sleep considerably during the presymptomatic state $(1.5 \pm 0.5 \mathrm{~min})$ but exhibited a strong increase in time spent sleeping in the symptomatic state ( $7.6 \pm 1.3 \mathrm{~min})$. For all monkeys, naps occurring during the light-OFF sessions were mainly composed of S1-S2. Interestingly, M1 had an appearance of a few sleep episodes of S3 and REM sleep and this from the presymptomatic state (Fig. 3.B).

Table 2

Sleep parameters obtained in light-OFF sessions for each animal M1, M2 and M3 in healthy, presymptomatic and symptomatic states.

\begin{tabular}{|c|c|c|c|c|c|c|c|c|c|}
\hline & \multicolumn{3}{|l|}{ M1 } & \multicolumn{3}{|l|}{ M2 } & \multicolumn{3}{|l|}{ M3 } \\
\hline & Healthy & Presymptomatic & Symptomatic & Healthy & Presymptomatic & Symptomatic & Healthy & Presymptomatic & Symptomatic \\
\hline $\mathrm{SL}(\min )$ & $\begin{array}{l}13.5 \pm \\
0.9\end{array}$ & $6.3 \pm 0.4$ * & $9.8 \pm 0.8$ * & $\begin{array}{l}11.6 \pm \\
0.8\end{array}$ & $7.4 \pm 1.1$ * & $6.3 \pm 1.1$ * & 20 & $16 \pm 1$ & $8.2 \pm 1.4$ * \# \\
\hline $\begin{array}{l}\text { S1-S2 } \\
(\mathrm{min})\end{array}$ & $\begin{array}{l}5.1 \pm \\
0.7\end{array}$ & $7.2 \pm 0.3$ * & $4.3 \pm 0.6$ & $\begin{array}{l}3.9 \pm \\
0.5\end{array}$ & $8.2 \pm 0.9$ * & $7 \pm 0.8$ * & 0 & $1.5 \pm 0.5$ & $6.7 \pm 1.2$ * \# \\
\hline S3 (min) & 0 & $0.6 \pm 0.2$ * & $0.1 \pm 0.1$ & 0 & 0 & 0 & 0 & 0 & 0 \\
\hline $\begin{array}{l}\text { REM } \\
\text { Sleep } \\
\text { (min) }\end{array}$ & 0 & $1.6 \pm 0.2$ * & $1 \pm 0.3$ * & 0 & 0 & 0 & 0 & 0 & 0 \\
\hline
\end{tabular}

Table 2: Sleep parameters obtained in light-OFF sessions for each animal in healthy, presymptomatic and symptomatic states expressed in mean values $( \pm$ SEM) derived from 30 minimum light-OFF sessions pooled together for each condition and animal. SL refer to the time in minute between the light turned off and the first sleep episode. Values in lines 2, 3 and 4 are expressed as the mean duration \pm SEM in min of each sleep stages (S1 and S2 pooled together, S3 and REM sleep). $\mathrm{P}<0.05$ * different from healthy state; \# different from presymptomatic state: Kruskal-Wallis test followed by Dunn's multiple comparisons test in the event of statistically significant differences.

\section{Discussion}

The progressive intoxication used in this study (4-6 months) allowed us to reproduce the slow evolution of PD with an early state characterized by no motor symptoms and no significant beta oscillations on the EEG. Based on these findings, this period can be considered similar to the presymptomatic state encountered in PD patients. During this period, the sleep architecture was significantly disorganized, with less intense but more S3 at the expense of S1 and $\mathrm{S} 2$, a phenomenon previously evoked in another NHP study ${ }^{28}$. As S3 seems to be relevant for the neuroprotective process and can be considered as a restorative sleep stage ${ }^{30,31}$, the added presence of S3 could reflect the implementation of some adaptive phenomena that would take place in the early stage of the disease $\mathrm{s}^{32}$ to counteract the decrease in the delta frequencies power. We also found an increase in the $9-12 \mathrm{~Hz}$ frequency during $\mathrm{S} 3$, which is the characteristic of slow sleep spindles ${ }^{33}$. Sleep spindles are bursts of oscillatory brain activity with thalamic origin and known to have a role in sleep maintenance ${ }^{34}$. Therefore, induced slow spindles during S3 can be regarded as an additional compensatory mechanism to protect sleep elicited by the delta

Page 5/13 
power loss. In addition, the power of EMG during REM sleep in the presymptomatic state tended to increase, this loss of atonia could also be considered as a premotor sign of PD. In connection with REM sleep, we also show a significant increase in heart rate variability in the presymptomatic stage characterized by an increase in the coefficient of variation of RR intervals while having a similar heart rate mean. These significant changes provide interesting prodromal tools for further analyses.

Additionally, during the daytime, several specific disturbances were identified. All animals exhibited difficulties waking up in the morning, which resulted in a very early first nap after wake, and continuous naps until the middle of the day. This altered wakefulness was also revealed by mMSLT which showed faster SL with longer sleep episodes. These W/S disturbances were well pronounced for M1 and M2 and less marked for M3. M3 was younger than the others, which can partly explain these differences. It is also relevant to mention that only M1 slept in REM sleep during the naps of this presymptomatic period; this phenomenon, typical of sleep attacks encountered in narcolepsy, is also described in PD patients. These discrepancies between the animals are in accordance with the variability of PD expression in humans ${ }^{35}$. Interestingly, some studies carried out in de novo patients have shown that sleep quality is not altered, although daytime sleepiness is perceptible ${ }^{36,37}$. Our results also showed this discrepancy, which could be due to the early alteration of systems related to wakefulness, more than those of sleep, which would not be sufficiently active to maintain great wakefulness.

Then, in stable parkinsonians NHPs, we observed drastic and significant changes during both nighttime and daytime. Indeed, during nighttime, we observed a significant decrease in S3 and REM sleep, a significant increase in WASO and a dramatic decrease in SE, as already described ${ }^{28,29}$ and observed in patients in advanced $\mathrm{PD}^{38,39}$. Among the most common sleep disorders, patients very often present REM sleep behavior disorders, which are REM sleep parasomnias leading to abnormal vocalizations and abnormal behavior ${ }^{40}$. In our study, no abnormal or violent behavior was observed, which is in accordance with other NHP studies ${ }^{28,29}$. However, we described a significant loss of muscular atonia during REM sleep in parkinsonian animals, which is also observed in marmosets ${ }^{41}$ and parkinsonian patients (Arnulf 2012). Even if this event is less marked in NHPs than in patients, it suggests an alteration of mechanisms involved in atonia ${ }^{43}$. Dopamine dysregulation can modify the function of brainstem nuclei, such as the locus cœruleus alpha or the pedunculopontine nucleus that are known to regulate atonia during REM sleep ${ }^{40}$.

During daytime, once stable parkinsonian syndrome was installed, it was no longer possible to count the naps, which seemed to be diffused throughout the day. This finding are consistent with the state of permanent sleepiness encountered among parkinsonian patients. The onset of an increasingly early morning nap is reminiscent of a delayed phase syndrome, that is also reported in patients with $\mathrm{PD}^{44}$. Dopamine depletion could also induce disturbances of the circadian clock, which is mediated by a regulatory mechanism called process $\mathrm{C}^{45}$. In MPTP-NHPs, this fact has already been reported, but more as an inability of the circadian clock to efficiently drive rhythmic locomotor activity ${ }^{26}$. All these observations, especially the early onset of morning sleep while sleep efficiency remains stable in the presymptomatic state, are in agreement with human studies concluding that EDS may be a NMS that is unrelated to other sleep disorders ${ }^{3,46}$. Furthermore, several studies have demonstrated a correlation between EDS and dopaminergic treatment ${ }^{47,48}$. In our study, we did not administer dopaminergic treatment, which showed that EDS in MPTP-treated NHPs is not necessarily associated with drugs and that it can be due to the early deregulation of dopaminergic and non-dopaminergic systems.

This study provides a solid documentation of the clinical evolution of motor and NMSs, but we are aware that it does not allow us to follow the degeneration of different brain structures; therefore, we cannot conclude upon the involvement of one or more neurotransmitters. Nevertheless, it offers a relevant model of the disease progression that can be used as a basis for the investigation of neuropathological mechanisms and can help in the management of human pathology. Our study demonstrates significant prodromal W/S disorders in NHP progressive model of PD expressed as disorganization of the nighttime-sleep and daytime-nap architectures. The increase of EDS is a sign easily detectable by the patient himself and deserves to be taken into consideration by the clinicians. This first sign may lead the clinicians to carry out more detailed polysomnographic analyses which will make it possible to show a complete deregulation of the W/S behavior with S3 abnormalities. These biomarkers could be very crucial for the identification of patients who are at risk of developing PD and could allow the delineation of a therapeutic window for the development of early neuroprotective strategies.

\section{Materials And Methods}

Animals. In accordance with the policy of Grenoble Alpes University and the Grenoble Institut of Neurosciences (B3851610008) and with French legislation, experiments were performed in compliance with the European Community Council Directive of 2010 (2010/63/UE) for care of laboratory animals and the ARRIVE guidelines. All procedures were reviewed and validated by the "Comité éthique du GIN n004" and was authorized by the Direction Départementale des Services Vétérinaires de l'Isère - Ministère de l'Agriculture et de la Pêche, France. We used three male adult monkeys (Macaca fascicularis - Mauritius) weighed 6-10kg and were 5-10 years. Animals were kept under controlled conditions, $12 \mathrm{~h}$ light/dark cycles, $23 \pm 2{ }^{\circ} \mathrm{C}$, and $50 \pm 5 \%$ humidity. Animals were pair housed, had access ad libitum to food and water and supplemental fresh fruit was given once a day.

Apparatus. Monkeys were implanted with a polysomnographic equipment, a radio-telemeter transmitter (D70-EEE, Data Science International, France) for longterm recording in freely moving animals. The transmitter had three channels biopotential for recording electroencephalogram (EEG), electro-oculogram (EOG), and electromyogram (EMG) signals with a sampling rate of $500 \mathrm{~Hz}$ and a gain of 75 and actimetry count. Signals were acquired via two receivers mounted on the home cage and behavioral cage and then forwarded to a data exchange matrix connected to a computer for data storage and off-line analysis.

Surgery. The surgery was performed under aseptic conditions and general anesthesia. Animals were anesthetized with Ketamine (7mg/kg, i.m.) and Xylazine $\left(0.6 \mathrm{mg} / \mathrm{kg}\right.$, i.m.) then intubated and switched to isoflurane mixed with oxygen. Animals were spontaneously breathing. Respiration rate, Et- $\mathrm{CO}_{2}$ and $\mathrm{O}_{2}$ saturation were monitored with a Comdek MD-660P monitor. Saline solution ( $\mathrm{NaCl} 0.9 \%)$ was infused intravenously all along the surgery for drug access and hydration. Analgesic therapy (Ketoprofen $2 \mathrm{mg} / \mathrm{kg}$ i.m.) were provided during one-week post-operative period. The radio-telemeter transmitter was implanted within a subcutaneous pocket in their back and the electrode leads were tunneled to the skull. EEG was recorded using two electrodes screwed unilaterally 
(one frontal and one parietal) into the skull, EOG was acquired from two electrodes affixed unilaterally at the orbital arch bone (one at the top and one at the external side) and EMG was monitored from two leads sutured into the neck musculature. The reference was fixed on the skull at the left occipital level.

MPTP treatment. Monkeys were intoxicated by intramuscular injection of MPTP under light anesthesia (Ketamine 2-4mg/kg). A progressive protocol was used to obtain a presymptomatic phase, i.e. before the motor symptoms, consisting in small doses of MPTP $(0.2-0.5 \mathrm{mg} / \mathrm{kg}$, in NaCl $0.9 \%)$ at two-weeks interval until the parkinsonian symptoms were stable. M1 received 18 injections ( $7.55 \mathrm{mg} / \mathrm{kg}$ total), M2 received 8 injections ( $2.2 \mathrm{mg} / \mathrm{kg}$ total) and $\mathrm{M} 3 \mathrm{received} 18$ injections $(6.1 \mathrm{mg} / \mathrm{kg}$ total). All animals had comparable state of stable parkinsonism.

Motor score. The severity of parkinsonism was evaluated before, during and after MPTP intoxication in the home cage, using a rating scale, combining the most recurring items from eight commonly used parkinsonism scales ${ }^{49}$. This scale includes eight clinical symptoms (general activity, frequency of each arms movements, posture, bradykinesia, tremor, feeding, freezing and vocalization), rated between 0 (normal) and 2-3 (maximal disability), with a total score out of 25 . Evaluations were performed by the same observer once a week at $2 \mathrm{pm}$ for $15 \mathrm{~min}$. The spontaneous activity was quantified by the implanted radiotelemeter transmitter and expressed in counts/min. Activity values are collected every 10 seconds, according to the changes of the signal perceived by the receivers mounted in the cage. If the animal is stationary the signal strength remains constant and equal to zero and if the animal moves the signal strength changes and is counted as movement.

DaTscan. In addition to this clinical motor evaluation, the first animal (M1) had an individual follow-up of the striatale dopaminergic system, all along the induction of the parkinsonian syndrome, which gives a global idea of the impact of MPTP on the dopamine degeneration. The fixation of the loflupane (123I) tracer, a radioligand with a high affinity for presynaptic dopamine transporters, was quantified in the striatum to observe the dopamine reduction. For all the exams, the animal was anesthetized and a dose of $90 \mathrm{MBq}$ was injected intravenously $3 \mathrm{~h}$ before the acquisition. Then, the images which had the highest radioactivity count, and the one above and below (three cuts) were selected and averaged. Regions of interest were drawn manually for each striatum and an area of occipital cortex served as a reference for the background noise (region of non-specific ${ }^{123}$ I uptake). The final activity count was determined as the ratio of striatal activity to occipital activity.

Sleep data analysis. Sleep scoring was performed offline on a software (NeuroScore, Data Science international). EEG and EOG was bandpass-filtered in the range of 0.3 to $35 \mathrm{~Hz}$ and $E M G$ was bandpass-filtered in the range of 10 to $100 \mathrm{~Hz}$. Sleep stages were manually determined according to the American Academy of Sleep Medicine criteria and performed in 30 s epoch $^{50}$. Different stages were identified: active wake (A), quiet wake (W), and non-REM sleep: light sleep stage 1 (S1) and stage 2 (S2), deep sleep stage 3 (S3) and REM sleep (R) (Fig. 4). Movement and chewing artefacts were mostly produced during active wake and were correlated with the simultaneous video observation. EEG power spectral analyses were performed for all scored 30 s epochs, and for the different stages of wakefulness and sleep, to verify whether the visual scoring is consistent with the expected frequency bands (Fig. 4). All epochs with artefacts were excluded from spectral analysis.

EMG and EEG spectral analysis. Power spectral density analysis was performed on EMG signals specifically during active wakefulness and REM sleep and on EEG signals specifically during active wakefulness and deep sleep S3. This analysis was performed in healthy, presymptomatic, and symptomatic states to observe any changes between these different conditions. Thus, 300 samples (100 samples for each animal) from random 30 s epochs for these different wake and sleep stages and in the different conditions were analyzed. For each 30 s epoch, an estimate of the power spectral density was computed using the Fast Fourier Transform and Hamming windowing technique and averaged (Matlab). Data are expressed in mean $\pm 95 \%$ confidence interval.

Nighttime sleep quality evaluation. Nighttime sleep data were analyzed from $7 \mathrm{pm}$ to $7 \mathrm{am}$. Two $12 \mathrm{~h}$ recording sessions per week were performed until a total of ten recordings were reached at minimum, in each experimental condition. For each $12 \mathrm{~h}$ period of nighttime, the sleep latency (SL) (min), total sleep time (TST) (min), duration of each stage (\%), wake time after sleep onset (WASO) (min) and sleep efficiency (expressed in \%, as the ratio of TST to the $12 \mathrm{~h}$ nocturnal time) were calculated.

Daytime hypersomnia evaluation. Assessment of hypersomnia was done by long-term recording of $12 \mathrm{~h}$ from $7 \mathrm{am}$ to $7 \mathrm{pm}$. Two $12 \mathrm{~h}$ recording sessions per week were performed until a total of ten recordings were reached at minimum, in each experimental condition. For each $12 \mathrm{~h}$ period of daytime, the first sleep after wake, the TST and the duration of each stage (\%) were calculated.

Daytime sleepiness evaluation. Daytime sleepiness was evaluated using a modified multiple sleep latency test (mMSLT), performed $2 \mathrm{~h}$ after waking up. In quiet room, the lights were turned off 3 times for a duration of 20min, $1 \mathrm{~h}$ apart (light-OFF sessions at 10 am, 11 am and 12 am). mMSLT was performed twice a week in healthy state until a minimum of ten experimentations were acquired and all along the parkinsonian syndrome induction for a minimum of ten experimentations in presymptomatic and symptomatic state. SL was determined if a 30s epoch of scorable sleep was observed. If no sleep onset was observed, SL was designated to be $20 \mathrm{~min}$ as used in human and NHP studies ${ }^{51,52}$. The two wake stages A and W were pooled together and the two light sleep stages S1 and S2 were pooled together. The following parameters were calculated for each 20min period of light-OFF session: SL (min), duration of wake and sleep (min).

REM sleep heart rate variability analysis. 12 epochs of $2 \mathrm{~min}$ of EMG during REM sleep were randomly pick up including the perception of the cardiac R peak which gives us an electrocardiogram-like. For each epoch, beat-to-beat (RR) variability was analysed in the time domain including the mean RR (meanNN) interval and its standard deviation (StdNN) and the coefficient of variation of RR intervals |[StdNN/MeanNN] x 100| showing the relative extent of the data. Poincaré plot, which portrays the relationship between successive $R R$ intervals $\left(R R_{n+1}\right.$ interval plotted against the preceding one $\left.R R_{n}\right) w_{a s}$ done. This plot provides summary information about RR interval and density distribution which are considered as general measures of heart rate variability.

Statistical analysis. Standard statistical methods using GraphPad Prism 8 were applied. A Kruskal-Wallis test followed by Dunn's multiple comparisons test was used for the comparison of sleep parameters at healthy, presymptomatic and symptomatic states. Data are presented as mean \pm standard error of the

Page $7 / 13$ 
mean (SEM) and the statistical significance was considered at a probability (p) value $\leq 0.05$.

\section{Declarations}

\section{Acknowledgments and funding sources}

The authors would address their thanks to Luc Loveiko from CHUGA and also Jessy Hugues Dit Ciles and Clarice Froget for their technical and animal care assistance. This study was supported by CEA Clinatec Grenoble, the Fondation de l'Avenir Paris ( $\mathrm{n}^{\circ}$ AP-RMA-2015-002) and the Fondation de France Paris, France $\left(n^{\circ}\right.$ 00086316). Data were acquired on a platform of France Life Imaging network partly by the grant "ANR-11-INBS-0006". Sponsors had no role in the study design, data collection, data analysis or data interpretation.

\section{Author Contributions}

Aurélie Davin: Acquisition and analysis of data; Statistical analysis; Manuscript preparation (writing the first draft and figures)

Stéphan Chabardès: Manuscript preparation (review and critique)

Hayat Belaid: Research project (surgery)

Daniel Fagret and Loic Djaileb: Research project (DatScan execution)

Yves Dauvilliers: Manuscript preparation (review and critique)

Olivier David: Manuscript preparation (review and critique)

Napoléon Torres-Martinez: Manuscript preparation (review and critique)

Brigitte Piallat: Conception and design of the study; Manuscript preparation (writing the first draft)

\section{Competing of Interest}

Nothing to report.

\section{References}

1. Abbott, R. D. et al. Excessive daytime sleepiness and subsequent development of Excessive daytime sleepiness and subsequent development of Parkinson disease. Neurology 65, 1442-1446 (2005).

2. Yoo, S.-W., Kim, J.-S., Oh, Y.-S., Ryu, D.-W. \& Lee, K.-S. Excessive daytime sleepiness and its impact on quality of life in de novo Parkinson's disease. Neurol Sci 40, 1151-1156 (2019).

3. Arnulf, I., E. Konofal, Merino-Andreu, L., Houeto, J. L. \& V. Mesnage. Parkinson's disease and sleepiness An integral part of PD. Neurology 58, 1019-1024 (2002).

4. Bargiotas, P., Schuepbach, M. W. M. \& Bassetti, C. L. Sleep - wake disturbances in the premotor and early stage of Parkinson 's disease. Curr Opin 41, 763-772 (2016).

5. Cock, V. C. De \& Arnulf, I. REM sleep behavior disorders and their characteristics in Parkinson 's disease. Rev Neurol (Paris) 164, 683-691 (2008).

6. Cock, C. De et al. Daytime Sleepiness in Parkinson 's Disease: A Reappraisal. PLoS One 9, 4-10 (2014).

7. Ghorayeb, I. et al. A nationwide survey of excessive daytime sleepiness in Parkinson's disease in France. Mov Disord 22, 1567-1572 (2007).

8. Ondo, W. G. et al. Daytime sleepiness and other sleep disorders in Parkinson's disease. Neurology 57, 1392-1397 (2001).

9. Schrempf, W., Brandt, M. D., Storch, A. \& Reichmann, H. Sleep Disorders in Parkinson 's Disease. Parkinsons Dis 4, $211-221$ (2014).

10. Breen, D. P., Vuono, R., Nawarathna, U. \& Fisher, K. Europe PMC Funders Group Sleep and Circadian Rhythm Regulation in Early Parkinson Disease. JAMA Neurol 71, 589-595 (2014).

11. Paredes-Rodriguez, E., Vegas-Suarez, S., Morera-Herreras, T., De Deurwaerdere, P. \& Miguelez, C. The Noradrenergic System in Parkinson's Disease. Front Pharmacol 11, 1-13 (2020).

12. Hirsch, E. C., Graybielt, A. N. N. M., Duyckaertst, C. \& Javoy-agid, F. Neuronal loss in the pedunculopontine tegmental nucleus in Parkinson disease and in progressive supranuclear palsy. PNAS 84, 5976-5980 (1987).

13. Fronczek, R. et al. Hypocretin (orexin) loss in Parkinson's disease. Brain 130, 1577-1585 (2007).

14. Thannickal, T. C., Lai, Y. Y. \& Siegel, J. M. Hypocretin (orexin) cell loss in Parkinson's disease. Brain 130, 1586-1595 (2007).

15. Postuma, R. B. \& Berg, D. Prodromal Parkinson 's Disease: The Decade Past, the Decade to Come State of the Field 10 Years Ago Key Advances in the Last Decade. Mov Disord 34, 665-675 (2019).

16. Bonnet, M. H. \& Arand, D. L. Heart rate variability: Sleep stage, time of night, and arousal influences. Electroencephalogr Clin Neurophysio/ $102,390-396$ (1997).

17. Bugalho, P. et al. Heart rate variability in Parkinson disease and idiopathic REM sleep behavior disorder. Clin Auton Res 28, 557-564 (2018).

18. Videnovic, A. et al. Circadian melatonin rhythm and excessive daytime sleepiness in Parkinson disease. JAMA Neuro/ 71, 463-469 (2014). 
19. Mendelsohn, A. R. \& Larrick, J. W. Sleep Facilitates Clearance of Metabolites from the Brain: Glymphatic Function in Aging and Neurodegenerative Diseases. Rejuvenation Res 16, 518-523 (2013).

20. Xie, L. et al. Sleep drives metabolite clearance from adult brain. 342, 1-11 (2013).

21. Vyazovskiy, V. V. Sleep, recovery, and metaregulation: Explaining the benefits of sleep. Nat Sci Sleep 7, 171-184 (2015).

22. Elkhenany, H., AlOkda, A., El-Badawy, A. \& El-Badri, N. Tissue regeneration: Impact of sleep on stem cell regenerative capacity. Life Sci 214, 51-61 (2018).

23. Blesa, J., Trigo-Damas, I., del Rey, N. L. G. \& Obeso, J. A. The use of nonhuman primate models to understand processes in Parkinson's disease. J Neural Transm 125, 325-335 (2018).

24. Emborg, M. E. Nonhuman Primate Models of Parkinson's Disease. ILAR J 48, (2007).

25. Choudhury, G. R. \& Daadi, M. M. Charting the onset of Parkinson-like motor and non-motor symptoms in nonhuman primate model of Parkinson's disease. PLoS One 13, e0202770 (2018).

26. Fifel, K. et al. Alteration of Daily and Circadian Rhythms following Dopamine Depletion in MPTP Treated Non-Human Primates. PLoS One 9, (2014).

27. Almirall, H. et al. Nocturnal sleep structure and temperature slope in MPTP treated monkeys. J Neural Transm 106, 1125-1134 (1999).

28. Barraud, Q. et al. Sleep disorders in Parkinson's disease: The contribution of the MPTP non-human primate model. Exp Neurol 219, 574-582 (2009).

29. Belaid, H. et al. Sleep Disorders in Parkinsonian Macaques: Effects of L -Dopa Treatment and Pedunculopontine Nucleus Lesion. J Neu 34, 9124-9133 (2014).

30. Rasch, B. \& Born, J. About sleep's role in memory. Physiol Rev 93, 681-766 (2013).

31. Dijk, D. J. Regulation and functional correlates of slow wave sleep. J Clin Sleep Med 5, S6-15 (2009).

32. Blesa, J. et al. Compensatory mechanisms in Parkinson's disease: Circuits adaptations and role in disease modification. Exp Neuro/ 298, 148-161 (2017).

33. Perl, O. et al. Odors enhance slow-wave activity in non-rapid eye movement sleep. J Neurophysio/ 115, 2294-2302 (2016).

34. Dang-Vu, T. T. et al. Interplay between spontaneous and induced brain activity during human non-rapid eye movement sleep. Proc Natl Acad Sci U S A 108, 15438-15443 (2011).

35. Wolters, E. C. Variability in the clinical expression of Parkinson's disease. J Neurol Sci 266, 197-203 (2008).

36. Brunner, H. et al. Microstructure of the non-rapid eye movement sleep electroencephalogram in patients with newly diagnosed Parkinson's disease: Effects of dopaminergic treatment. Mov Disord 17, 928-933 (2002).

37. Kaynak, D., Kiziltan, G., Kaynak, H., Benbir, G. \& Uysal, O. Sleep and sleepiness in patients with Parkinson's disease before and after dopaminergic treatment. Eur J Neurol 12, 199-207 (2005).

38. Yong, M. H., Fook-Chong, S., Pavanni, R., Lim, L. L. \& Tan, E. K. Case control polysomnographic studies of sleep disorders in Parkinson's disease. PLoS One 6, 1-7 (2011).

39. Mayer, G., Jennum, P., Riemann, D. \& Dauvilliers, Y. Insomnia in central neurologic diseases - occurrence and management. Sleep Med Rev 15 , 369-378 (2011).

40. Boeve, B. F. REM sleep behavior disorder: Updated review of the core features, the REM sleep behavior disorder-neurodegenerative disease association, evolving concepts, controversies, and future directions. Ann N Y Acad Sci 1184, 15-54 (2010).

41. Verhave, P. S. et al. REM sleep behavior disorder in the marmoset MPTP model of early Parkinson disease. Sleep 34, 1119-1125 (2011).

42. Arnulf, I. REM sleep behavior disorder: Motor manifestations and pathophysiology. Mov Disord 27, 677-689 (2012).

43. Dauvilliers, Y. et al. REM sleep behaviour disorder. Nat Rev Dis Prim 4, (2018).

44. French, I. T. \& Muthusamy, K. A. A Review of Sleep and Its Disorders in Patients with Parkinson's Disease in Relation to Various Brain Structures. Front Aging Neurosci 8, 1-17 (2016).

45. Borbély, A. A., Daan, S., Wirz-Justice, A. \& Deboer, T. The two-process model of sleep regulation: A reappraisal. J Sleep Res 25, 131-143 (2016).

46. Liguori, C. et al. Daytime sleepiness may be an independent symptom unrelated to sleep quality in Parkinson's disease. J Neuro/ 266, 636-641 (2019).

47. Arnulf, I. Excessive daytime sleepiness in parkinsonism. Sleep Med Rev 9, 185-200 (2005).

48. Chaudhuri, K. R., Pal, S. \& Brefel-Courbon, C. 'Sleep attacks' or 'unintended sleep episodes' occur with dopamine agonists: Is this a class effect? Drug Saf 25, 473-483 (2002).

49. Imbert, C., Bezard, E., Guitraud, S., Boraud, T. \& Gross, C. E. Comparison of eight clinical rating scales used for the assessment of MPTP-induced parkinsonism in the Macaque monkey. J Neurosci Methods 96, 71-76 (2000).

50. Iber, C., Ancoli-Israel, S., Chesson, A. \& Quan, S. The AASM Manual for the Scoring of Sleep and Associated Events: Rules, Terminology and Technical Specifications. American Academy of Sleep Medecine 1st ed.: W, (2007).

51. Littner, M. R. et al. Practice parameters for clinical use of the multiple sleep latency test and the maintenance of wakefulness test. Sleep 28 , 113-121 (2005).

52. Daley, J. T., Turner, R. S., Freeman, A., Bliwise, D. L. \& Rye, D. B. Prolonged assessment of sleep and daytime sleepiness in unrestrained macaca mullata. Sleep 29, 221-231 (2006).

\section{Figures}


(A)

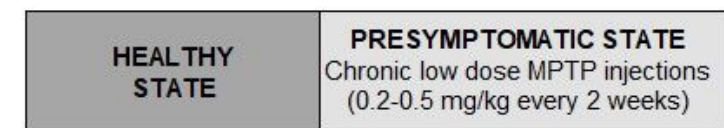

Score of

(B) parkinsonism
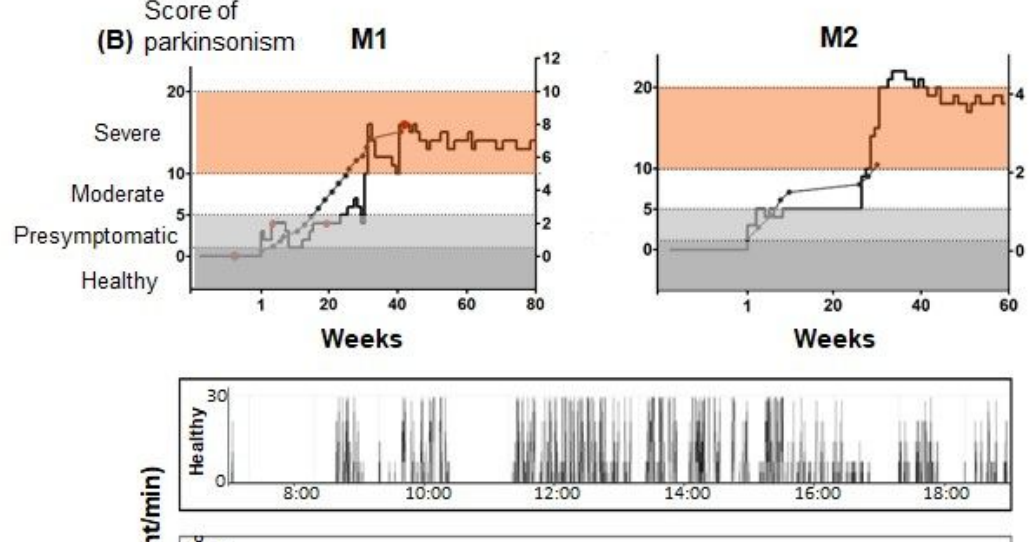

(C)
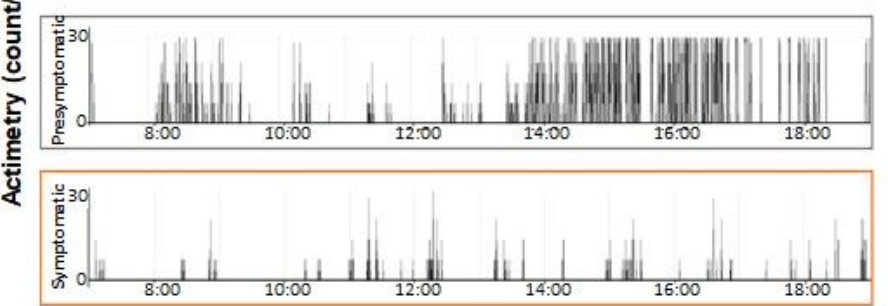

\section{(D)}

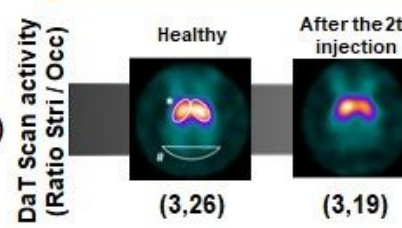

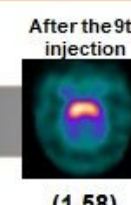

$(1,58)$

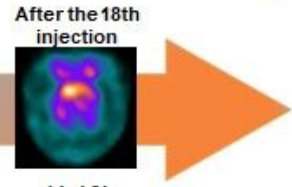

$(1,16)$
STABLE SYMPTOMATIC STATE
M3

Cumulative dose of MPTP $(\mathrm{mg} / \mathrm{kg})$
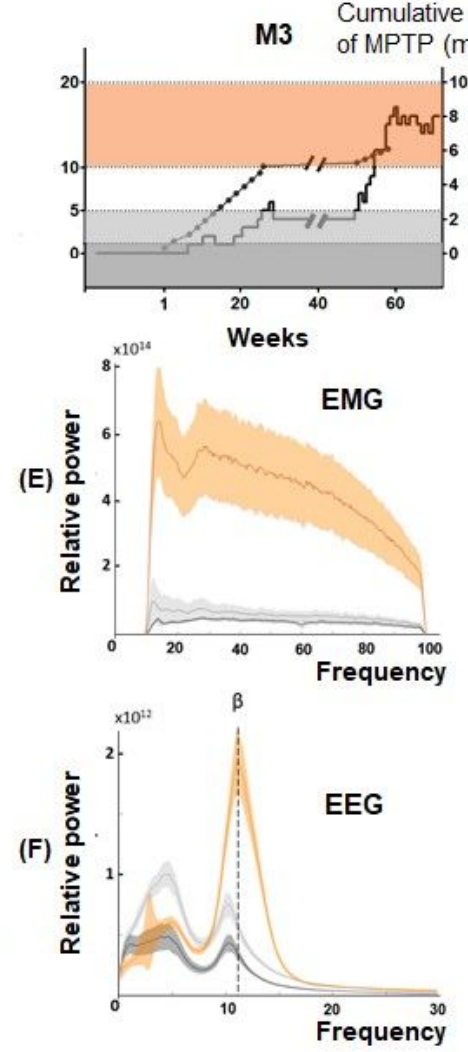

\section{Figure 1}

Panel of graphs showing the motor symptoms evaluation. (A) Design study and mean time of each periods for healthy (dark grey), presymptomatic (light grey) and symptomatic (orange) states with 8 weeks in mean of stabilization. (B) Longitudinal progression of Parkinson's disease for M1, M2 and M3, induced by injection of chronic low doses of MPTP, based on weekly observations. Solid black line shows the score of parkinsonism, gray line with dot shows the cumulative dose of MPTP where each dot corresponds to an injection of 0.2-0.5 mg/ $\mathrm{kg}$. The red dots on M1 graph correspond to the DaTscan imaging. The line break on M3 graphs correspond to a 2-month break due to infection problems, not taken into account in the analyses. (C) Actimetry expressed in count/min across different stages of the disease from 7 am to $7 \mathrm{pm}$. (D) Study of the uptake of the loflupane tracer (I-123) in M1, by DaTscan nuclear imaging examination, according to the different stages of the disease. *Striatum analysis area for activity measurement; \# cerebral background noise measurement. Note the asymmetric pattern against the left side from the 2nd injection of MPTP, which persists with the progression of the disease, and the progressive increased in cerebral background noise which indicates a difficulty of tracer fixation by lack of dopamine transporters. The ratio striatal/occipital activity is in parentheses under each image. (E) EMG spectral power analysis during active wake across different stages of parkinsonism in NHP; healthy in dark grey, presymptomatic in light grey and symptomatic in orange, expressed in mean $\pm 95 \%$ confidence interval. (F) EEG spectral power analysis during active wake across different stages of parkinsonism in NHP with the significant appearance of low-beta activity in symptomatic state. 

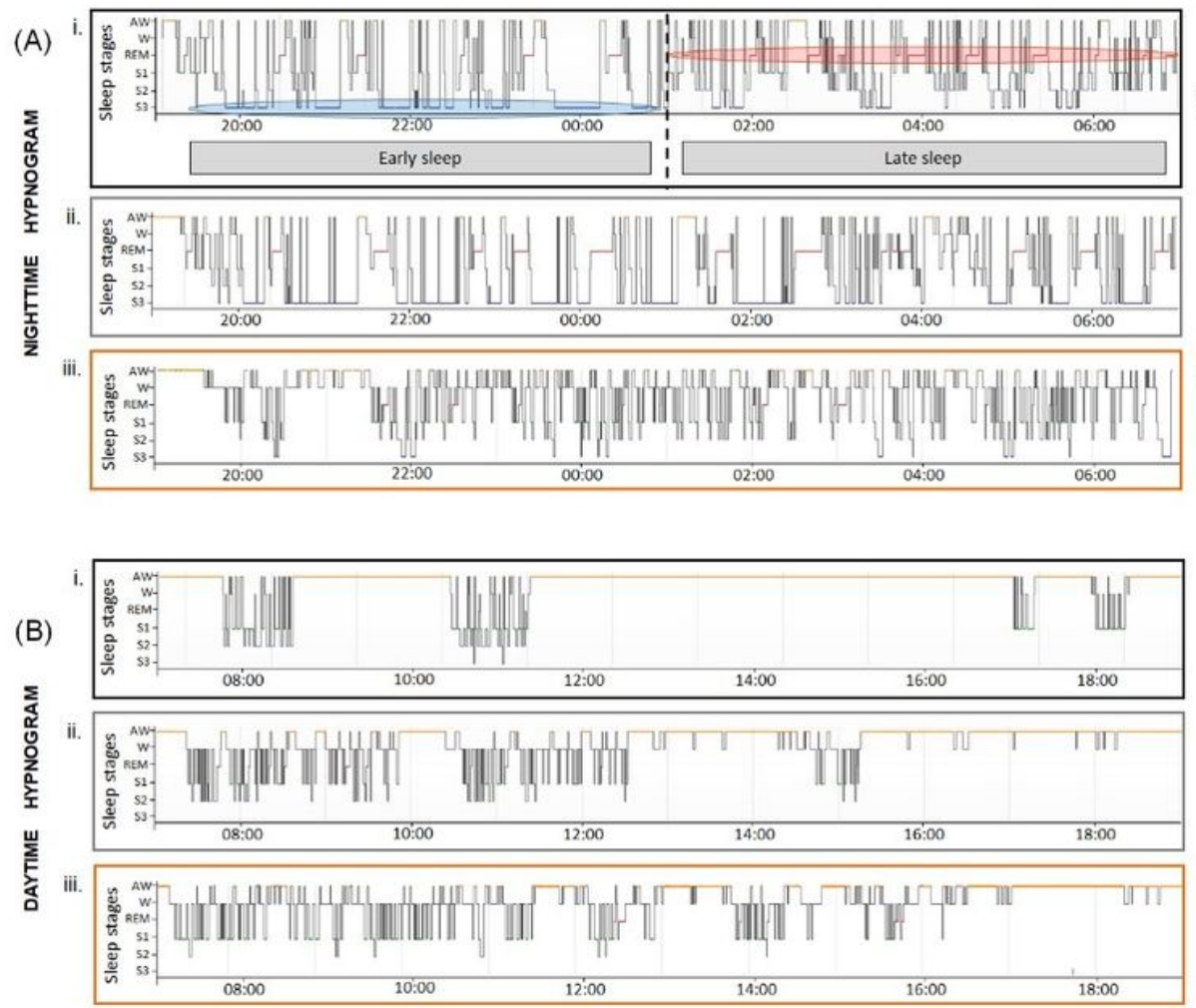
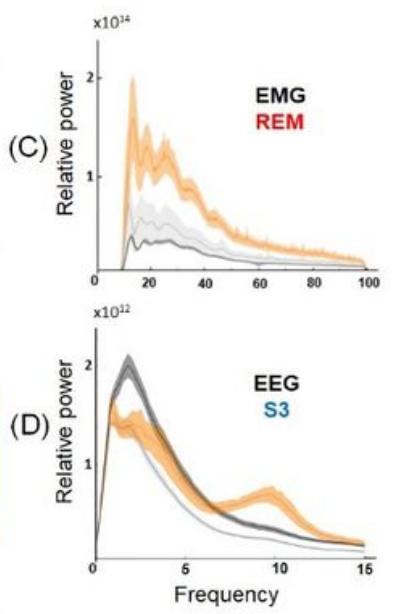

(E) ECG REM
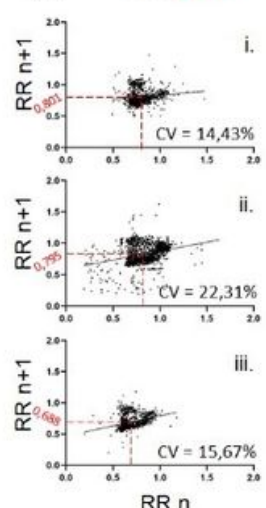

RR n

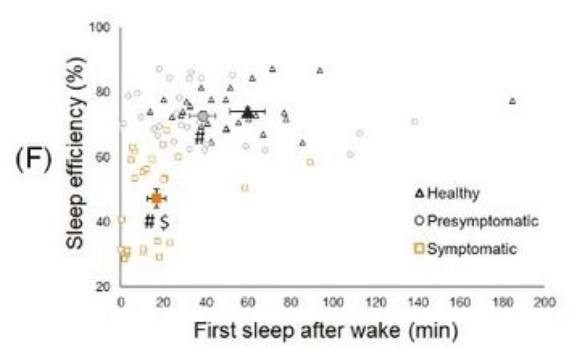

Figure 2

(A) Nighttime hypnogramm (from M1), in healthy (i), presymptomatic (ii) and symptomatic (iii) states. The early sleep period is mostly composed by S3 (circled in blue) and the late sleep period is mostly composed by REM sleep (circled in red). Note the sleep fragmentation in symptomatic state where S3 and REM sleep are almost inexistent in early sleep and late sleep period respectively. (B) Daytime hypnogramm (from M1), in healthy (i), presymptomatic (ii) and symptomatic (iii) states. Note the naps disorganization since the presymptomatic state and it is worse in symptomatic state. For both, (A) and (B) the line position indicates the sleep stage represented in $y$-axis. With $A=$ active wake; $W=$ quiet wake; 1,2,3= non-REM sleep stage 1, 2 and 3; $R=R E M$ sleep. (C) Mean relative power $\pm 95 \%$ confidence interval depending on frequency of EMG during REM sleep for the healthy (dark grey), the presymptomatic (light grey) and the symptomatic (orange) states. (D) Mean relative power $\pm 95 \%$ confidence interval depending on frequency of EEG during deep sleep S3 for the healthy (dark grey), the presymptomatic (light grey) and the symptomatic (orange) states. (E) Poincaré plots build from successive RR intervals expressed in second during REM sleep for the healthy (i), presymptomatic (ii) and symptomatic (iii) states. The red number correspond to the mean RR interval and coefficient of variation to the coefficient of variation. (F) Correlation between the daytime first sleep after wake (min) and the nighttime sleep efficiency (\%) for all animals in healthy, presymptomatic and symptomatic states. All the dots represent the latency of the first sleep after wake on the abscissa expressed in minute and the percentage of sleep efficiency on the ordinate. The mean and SEM are presented for each state. Kruskal-Wallis test followed by Dunn's multiple comparisons test with \# SL different from healthy and \$ sleep efficiency different from healthy. 
$\underline{\mathrm{M} 1}$

(A)

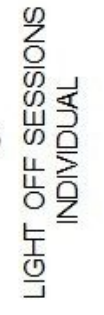

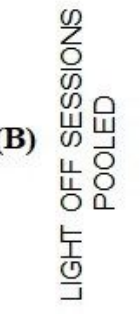

$\underline{\mathrm{M} 2}$

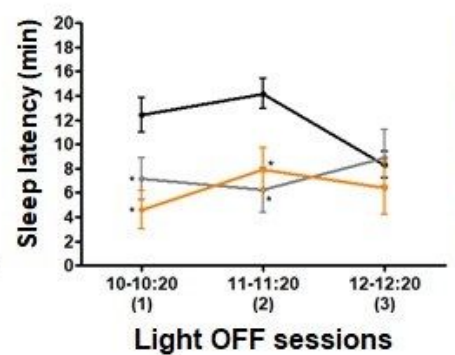

Light OFF sessions
$\underline{\mathrm{M} 3}$

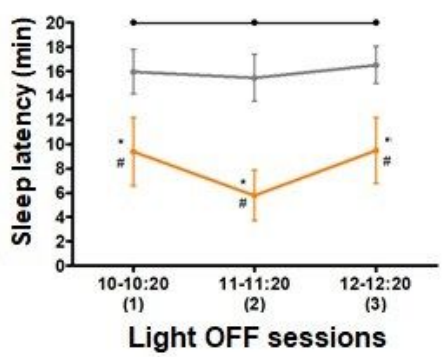

Light OFF sessions
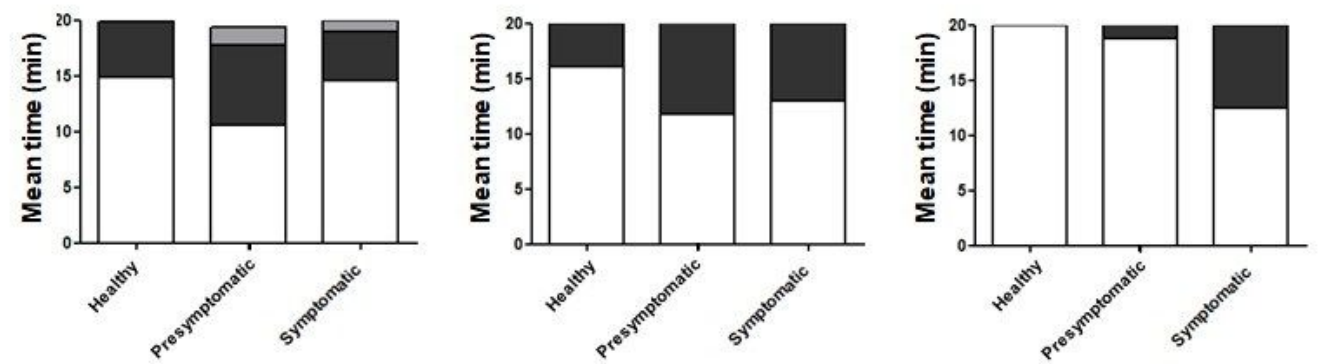

Figure 3

Panel of different sleep parameters obtained in light-OFF sessions of each animal in healthy, presymptomatic and symptomatic states. (A) SL expressed in min for each light-OFF session (1) from 10 am to 10:20 am, (2) from 11 am to 11:20 am, (3) from 12 am to 12:20 am and for healthy (black line), presymptomatic (grey line) and symptomatic (orange line) states. (B) Mean time of wake time (white), non-REM sleep (S1-S2-S3) (black) and REM sleep (grey) for all light-OFF session pooled together in healthy, presymptomatic and symptomatic states. $P<0.05$ * different from healthy state; \# different from presymptomatic state: Kruskal-Wallis test followed by Dunn's multiple comparisons test in the event of statistically significant differences.
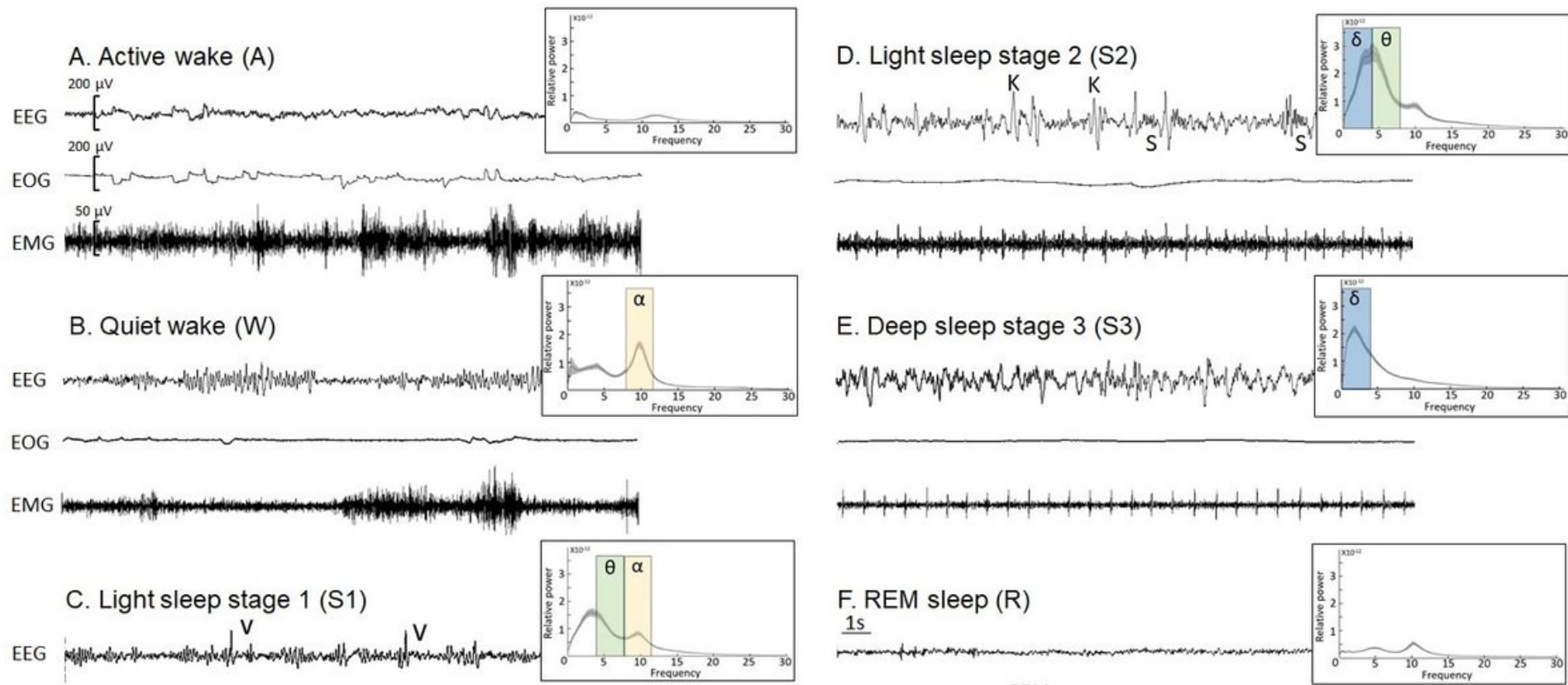

EOG

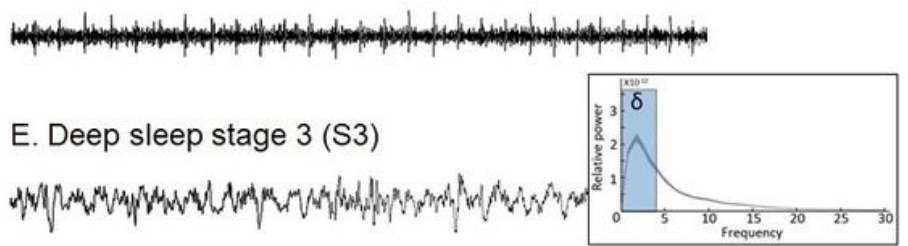

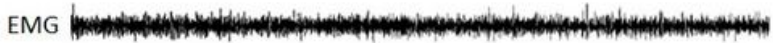

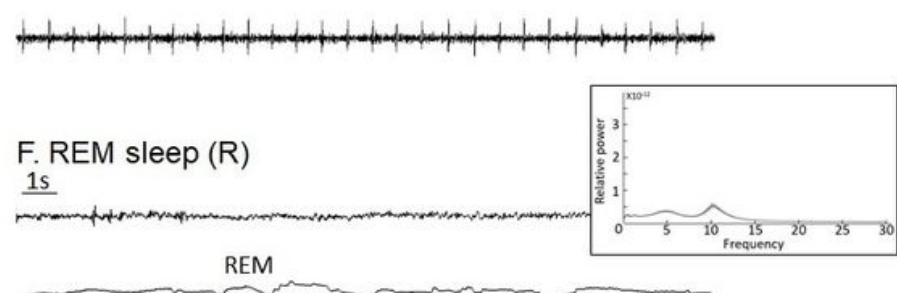

Figure 4

Polysomnographic recordings for Wake/Sleep stages analysis.

Thirty seconds epochs showing (A) active wake state with fast and low-voltage electroencephalogram (EEG), high-amplitude electro-oculogram (EOG) and high electromyogram (EMG) activity, (B) quiet wake state with alpha waves (8-12Hz) on EEG, (C) light sleep stage 1 with mix-frequency (alpha and theta 
waves) and vertex sharp waves ( $\mathrm{V}$ ) on EEG and reduced muscle tones (D) light sleep stage 2 with predominant theta waves (4-8Hz), spindles (S) and $\mathrm{K}$ complex (K) on EEG, absence of EOG and regular muscle tone, $(\mathrm{E})$ deep sleep stage 3 with ample delta frequencies $(0.3-5 \mathrm{~Hz})$ on $\mathrm{EEG}$ and reduced muscle tone, and (F) REM sleep with sawtooth EEG activity, rapid-eyes movements (REM) on EOG and absence of muscle tone. Mean $\pm 95 \%$ confidence interval of the relative spectral power of the EEG during each wake and sleep stages is shown to the right of EEG signals. 